\title{
Ground-state energy and relativistic corrections for positronium hydride
}

\author{
Sergiy Bubin and Kálmán Varga \\ Department of Physics and Astronomy, Vanderbilt University, Nashville, Tennessee 37235, USA \\ (Received 28 February 2011; revised manuscript received 14 June 2011; published 19 July 2011)
}

\begin{abstract}
Variational calculations of the ground state of positronium hydride (HPs) are reported, including various expectation values, electron-positron annihilation rates, and leading relativistic corrections to the total and dissociation energies. The calculations have been performed using a basis set of 4000 thoroughly optimized explicitly correlated Gaussian basis functions. The relative accuracy of the variational energy upper bound is estimated to be of the order of $2 \times 10^{-10}$, which is a significant improvement over previous nonrelativistic results.
\end{abstract}

DOI: 10.1103/PhysRevA.84.012509

PACS number(s): 31.15.ac, 31.15.xt, 31.30.J-, 36.10.-k

\section{INTRODUCTION}

The interest in studies of small molecules where one or more nuclei are replaced with a positron was originally motivated by the pioneering works of Hylleraas and Ore [1] and Ore [2]. These works predicted the dynamical stability of the positronium molecule ( $\left.\mathrm{Ps}_{2}\right)$ and positronium hydride (HPs). Since then there has been a large number of theoretical works devoted to the study the properties of these two fundamental systems (for works on HPs see [3-19] and references therein), including the ground, metastable, and resonant states.

Along with the numerous theoretical studies, there has also been an array of experimental works attempting to produce and detect HPs. In 1992, Schrader et al. reported [20] the formation of positronium hydride in collisions between positrons and methane and gave an estimate of the binding energy, $1.1 \pm 0.2 \mathrm{eV}$. Recently, positronium physics and chemistry gained new impetus when the first experimental observation of the positronium molecule was reported $[21,22]$. At present it is being investigated whether more complicated positronium compounds, such as $\mathrm{Ps}_{n}$ may exist. Moreover, it is believed that under certain conditions one may obtain a Bose-Einstein condensate, which will permit the creation of a powerful $\gamma$-ray laser. Due to its very short wavelength this laser could be used to probe objects as small as atomic nuclei [23].

Despite advances in experimental techniques and invention of new methods that allow a rapid creation of a large number of positrons in the laboratory [24,25], the experiments on atoms and molecules containing positrons remain a very challenging task. Thus, theoretical calculations are indispensable in elucidating the electronic structure and predicting the properties of such systems. In fact, just like the theoretical prediction of the existence of the $\mathrm{Ps}_{2}$ molecule was followed by its experimental verification $[21,22]$, there are planned experiments to confirm recent theoretical predictions of excited states of positronic systems $[10,17,26]$.

Over the years the accuracy of the calculations has been getting progressively higher due to advances in computer hardware and development of more sophisticated computational approaches. At some point, in order to further improve theoretical predictions it becomes necessary to consider relativistic and QED effects. However, while the number of works where positronium hydride has been studied is quite large, essentially all of them have been performed at the nonrelativistic level of theory. The only work where relativistic effects in HPs have been considered is that of Yan and Ho [13].
One of the goals of the present study is to fill this gap and improve the results obtained in [13].

The positronium hydride belongs to a special class of Coulomb systems. It lies between the $\mathrm{H}_{2}$ molecule and the fully nonadiabatic $\mathrm{Ps}_{2}$ molecule, where both nuclei are replaced with $e^{+}$. Since the mass of one of the "nuclei" in HPs is the same as that of an electron, its motion cannot be considered slow. Therefore, the Born-Oppenheimer approximation cannot be used to separate the electronic and positronic degrees of freedom. Another distinct difference between HPs and $\mathrm{H}_{2}$ is that the latter has a very large number of bound states, while for the positronium hydride only one excited state (of unnatural parity) have been predicted $[17,18]$.

These positronic systems are also important testing grounds for various quantum mechanical methods. The highly accurate calculations available for these molecules can be used as benchmark test to compare the relative merit of different approaches. The connection between these molecules has also motivated various studies to explore the existence of similar systems. For example the stability of charged four particle systems containing two negatively and two positively charged particles has been studied in [27-33]. Recent success in the production of trapped antihydrogen atoms [34,35] has renewed interest in the interaction of matter with antimatter [36]. These problems can only be addressed by highly accurate quantum-mechanical calculations.

Accurate description of positronic systems is challenging for traditional quantum-chemical methods as the convergence of the wave function and energy is usually quite slow. In this work we employ the variational method in which the correlation in the motion of all particles is treated by expanding the wave function in terms of basis functions explicitly dependent on all interparticle coordinates.

\section{FORMALISM}

The nonrelativistic Hamiltonian of an $N$-particle Coulomb system in the laboratory reference frame reads (in atomic units)

$$
H_{\text {NONREL }}=-\sum_{i=1}^{N} \frac{1}{2 M_{i}} \nabla_{\mathbf{R}_{i}}^{2}+\sum_{i=1}^{N} \sum_{j>i}^{N} \frac{Q_{i} Q_{j}}{R_{i j}} .
$$

Here $\mathbf{R}_{i}, M_{i}, Q_{i}$ are the position, the mass, and the charge of the $i$ th particle, $\nabla_{\mathbf{R}_{i}}$ is the gradient with respect to $\mathbf{R}_{i}$, and $R_{i j}=$ $\left|\mathbf{R}_{j}-\mathbf{R}_{i}\right|$ are interparticle distances. In the case of positronium hydride $N=4$. We will assume that the first particle in our 
system is a proton (deuteron, triton, etc.), the second one is a positron, and the third and fourth particles are electrons. Thus, $M_{1}=1836.15267247,3670.4829654,5496.9215269$, $\infty$ (when the heavy nucleus is a proton, deuteron, triton, and ${ }^{\infty} \mathrm{H}$, respectively) and $M_{2}=M_{3}=M_{4}=1$. The numerical values of particle masses were taken from [37]). The charges of the particles are $Q_{1}=Q_{2}=1, Q_{3}=Q_{4}=-1$.

In practice, instead of coordinates $\mathbf{R}_{1}, \ldots, \mathbf{R}_{N}$ it is convenient to use some set of "internal" coordinates, such as the Jacobi coordinates, or simply place the reference frame at one of the particles, e.g., particle 1, as is done in this work. This automatically separates out the motion of the center of mass. Our new coordinates are defined as follows [38-40]:

$$
\mathbf{r}_{1}=-\mathbf{R}_{1}+\mathbf{R}_{2}, \mathbf{r}_{2}=-\mathbf{R}_{1}+\mathbf{R}_{3}, \ldots, \mathbf{r}_{n}=-\mathbf{R}_{1}+\mathbf{R}_{N},
$$

where $n=N-1$. We will also introduce the following notation: $q_{i}=Q_{i+1}, m_{i}=M_{i+1}, \mu_{i}=m_{0} m_{i} /\left(m_{0}+m_{i}\right)$, and $i=0, \ldots, n$. In the new coordinates and notations the nonrelativistic Hamiltonian can be written in a convenient matrix form:

$$
H_{\text {nonrel }}=-\nabla_{\mathbf{r}}^{\prime} \mathbf{M} \nabla_{\mathbf{r}}+\sum_{i=1}^{n} \frac{q_{0} q_{i}}{r_{i}}+\sum_{i=1}^{n} \sum_{j>i}^{n} \frac{q_{i} q_{j}}{r_{i j}} .
$$

Here the $3 n \times 3 n$ mass matrix $\mathbf{M}=M \otimes I_{3}$, where $I_{3}$ is the $3 \times 3$ identity matrix and $\otimes$ stands for the Kronecker product. The $n \times n$ matrix $M$ is defined as follows: the diagonal elements are $1 /\left(2 \mu_{1}\right), 1 /\left(2 \mu_{2}\right), \ldots, 1 /\left(2 \mu_{n}\right)$, while the off-diagonal elements are $1 /\left(2 m_{0}\right)$. The prime symbol denotes vector or matrix transpose. $3 n$-component position vector $\mathbf{r}$ and the gradient vector $\nabla_{\mathbf{r}}$ are

$$
\mathbf{r}=\left[\begin{array}{c}
\mathbf{r}_{1} \\
\mathbf{r}_{2} \\
\vdots \\
\mathbf{r}_{n}
\end{array}\right], \quad \nabla_{\mathbf{r}}=\left[\begin{array}{c}
\nabla_{\mathbf{r}_{1}} \\
\nabla_{\mathbf{r}_{2}} \\
\vdots \\
\nabla_{\mathbf{r}_{n}}
\end{array}\right]
$$

In order to solve the nonrelativistic problem with Hamiltonian (3) we use the variational method in which the wave function is expanded in terms of explicitly correlated Gaussian basis functions. It has been demonstrated by numerous previous applications in atomic, molecular, and nuclear physics (see, for example, [10-12,15,16,26,38,39,41-50] and references therein) that the method is capable of providing extremely accurate solutions for systems containing up to 6-8 particles.

The ground state of positronium hydride is a state with zero total orbital angular momentum. Therefore, it is desirable and convenient to employ basis functions of $S$ symmetry in the calculations. The $S$ Gaussians we use in this work have the following form:

$$
\phi_{k}=\exp \left[-\mathbf{r}^{\prime}\left(A_{k} \otimes I_{3}\right) \mathbf{r}\right]=\exp \left[-\mathbf{r}^{\prime}\left(L_{k} L_{k}^{\prime} \otimes I_{3}\right) \mathbf{r}\right] .
$$

In the above expression $A_{k}$ is a symmetric, positive definite $n \times n$ matrix of exponential parameters that are unique for each basis function. Since the exponential parameters are subject to extensive optimization it is advantageous to choose matrix $A_{k}$ as a Cholesky-factored product, $A_{k}=L_{k} L_{k}^{\prime}$. Such a representation allows to avoid any constrains on the values of the elements of $L_{k}$, while the elements of the original matrix $A_{k}$ must obey the positive definiteness constrains.

In the case of a four-particle problem, explicitly correlated basis functions (5) can also be written in a more conventional form using the laboratory frame coordinates:

$$
\begin{aligned}
\phi_{k}= & \exp \left[-\lambda_{12}^{(k)} R_{12}^{2}-\lambda_{13}^{(k)} R_{13}^{2}-\lambda_{14}^{(k)} R_{14}^{2}-\lambda_{23}^{(k)} R_{23}^{2}\right. \\
& \left.-\lambda_{24}^{(k)} R_{24}^{2}-\lambda_{34}^{(k)} R_{34}^{2}\right],
\end{aligned}
$$

where $\lambda_{i j}^{(k)}$ are certain parameters related to the elements of matrix $L_{k}$.

The total trial wave function of the system is a product of the spatial and spin parts and is antisymmetrized with respect to the permutations of the electrons. For the ground state of HPs it can be written as

$$
\psi=\alpha_{1} \alpha_{2}\left(\alpha_{3} \beta_{4}-\beta_{3} \alpha_{4}\right)\left(1+P_{34}\right) \sum_{k=1}^{\mathcal{K}} \mathrm{c}_{k} \phi_{k}\left(\mathbf{r} ; L_{k}\right) .
$$

Here $\mathcal{K}$ is the size of the basis, $\mathrm{c}_{k}$ are the coefficients of the expansion, numbers 1 through 4 refer to a particular particle and $P_{34}$ is an operator that permutes spatial coordinates of electrons. $\alpha$ and $\beta$ have their usual meaning of spin-up and spin-down state.

The minimization of the energy functional with respect to coefficients $c_{k}$ in expansion (7) yields the generalized secular equation,

$$
\left(\mathrm{H}-E_{\text {nonrel }} \mathrm{S}\right) \mathrm{C}=0,
$$

where $\mathrm{H}$ and $\mathrm{S}$ are the $\mathcal{K} \times \mathcal{K}$ Hamiltonian and overlap matrices, respectively. Upon solving (8) one finds the set of optimal coefficients $\mathbf{c}_{k}$ and the upper bounds to the nonrelativistic energies. In this work we only deal with the lowest energy solution.

Since HPs is a small system with unity particle charges, the relativistic as well as quantum electrodynamics (QED) effects in this system are small in magnitude and can be accounted for in the framework of the perturbation theory in which the nonrelativistic solution serves as the zeroth-order approximation. The total energy of the system is sought as a series in powers of the fine structure constant, $\alpha$ :

$$
E_{\mathrm{TOT}}=E_{\mathrm{NONREL}}+\alpha^{2} E_{\mathrm{REL}}^{(2)}+\alpha^{3} E_{\mathrm{QED}}^{(3)}+\ldots
$$

The successive corrections, $E_{\mathrm{REL}}^{(2)}, E_{\mathrm{QED}}^{(3)}$, and the higher order ones can be evaluated as the expectation values of some effective operators. In this work we consider only the leading relativistic corrections proportional to $\alpha^{2}$. The total Hamiltonian is then

$$
H_{\mathrm{TOT}}=H_{\mathrm{NONREL}}+\alpha^{2} H_{\mathrm{REL}},
$$

where the relativistic part, $H_{\mathrm{REL}}$, consists of several terms,

$$
H_{\mathrm{REL}}=H_{\mathrm{MV}}+H_{\mathrm{D}}+H_{\mathrm{OO}}+H_{\mathrm{SS}}+H_{\mathrm{A}},
$$

traditionally called the mass-velocity (MV), Darwin (D), orbit-orbit (OO), spin-spin (SS), and annihilation channel (A) corrections. In the general case, there is also the spin-orbit (SO) term present in the sum. However, its contribution vanishes for $S$ states. The explicit expressions for the corresponding 
operators in the laboratory coordinates are the following $[51,52]$ :

$$
\begin{gathered}
H_{\mathrm{MV}}=-\frac{1}{8} \sum_{i=1}^{N} \frac{1}{M_{i}^{3}} \nabla_{\mathbf{R}_{i}}^{4}, \\
H_{\mathrm{D}}=-\frac{\pi}{2} \sum_{j=1}^{N} \sum_{i \neq j}^{N} \frac{Q_{i} Q_{j}}{M_{i}^{2}} \delta\left(\mathbf{R}_{i j}\right), \\
H_{\mathrm{OO}}=\frac{1}{2} \sum_{j=1}^{N} \sum_{i>j}^{N} \frac{Q_{i} Q_{j}}{M_{i} M_{j}} \frac{1}{R_{i j}} \\
\times\left(\nabla_{\mathbf{R}_{i}} \cdot \nabla_{\mathbf{R}_{j}}+\frac{1}{R_{i j}^{2}} \mathbf{R}_{i j} \cdot\left(\mathbf{R}_{i j} \cdot \nabla_{\mathbf{R}_{i}}\right) \nabla_{\mathbf{R}_{j}}\right), \\
H_{\mathrm{SS}}=-\frac{8 \pi}{3} \sum_{i=1}^{N} \sum_{j>i}^{N} \frac{Q_{i} Q_{j}}{M_{i} M_{j}} \mathbf{S}_{i} \cdot \mathbf{S}_{j} \delta\left(\mathbf{R}_{i j}\right), \\
H_{\mathrm{A}}=-2 \pi \sum_{i=1}^{N} \sum_{j>i}^{N} \frac{Q_{i}}{M_{i} M_{j}}\left(\frac{3}{4}+\mathbf{S}_{i} \cdot \mathbf{S}_{j}\right) \delta\left(\mathbf{R}_{i j}\right) .
\end{gathered}
$$

In the above expressions, $\delta\left(\mathbf{R}_{i j}\right)$ stands for the threedimensional Dirac $\delta$ function and $\mathbf{S}_{i}$ denotes the spin of the $i$ th particle. Notation $i \in$ leptons in formula (13) means that index $i$ runs over leptons only (in our case two electrons and a positron). In the general case it is also possible to include other types of particles (if there are any) in that expression. It is particularly straightforward for spin- $1 / 2$ fermions where the corresponding $g$ factors need to be used. However, since all other particles have masses larger by several orders of magnitude than that of the electron, their contribution will be very tiny and much smaller than the QED corrections, which we do not consider in this work. For this reason we use the expression given in (13). In formula (16) the double sum runs over electron-positron pairs only, i.e., only those terms should be included where one of the indices $i, j$ belongs to an electron and the other one to a positron.

Upon transforming from the laboratory frame coordinates to the internal ones the expressions for the relativistic operators become (as before, we use lower case letters to refer to objects in the internal frame):

$$
\begin{aligned}
H_{\mathrm{mv}}= & -\frac{1}{8}\left[\frac{1}{m_{0}^{3}}\left(\sum_{i=1}^{n} \nabla_{\mathbf{r}_{i}}\right)^{4}+\sum_{i=1}^{n} \frac{1}{m_{i}^{3}} \nabla_{\mathbf{r}_{i}}^{4}\right], \\
H_{\mathrm{d}}= & -\frac{\pi}{2}\left[\sum_{\substack{i=1 \\
i \in \text { leptons }}}^{n} \frac{q_{0} q_{i}}{m_{0}^{2}} \delta\left(\mathbf{r}_{i}\right)+\sum_{\substack{i=1 \\
i \in \text { leptons }}}^{n} \frac{q_{0} q_{i}}{m_{i}^{2}} \delta\left(\mathbf{r}_{i}\right)\right. \\
& \left.+\sum_{j=1}^{n} \sum_{\substack{i \neq j \\
i \in \text { leptons }}}^{n} \frac{q_{i} q_{j}}{m_{i}^{2}} \delta\left(\mathbf{r}_{i j}\right)\right],
\end{aligned}
$$

$$
\begin{aligned}
H_{\mathrm{oo}}= & -\frac{1}{2} \sum_{i=1}^{n} \sum_{j=1}^{n} \frac{q_{0} q_{j}}{m_{0} m_{j}}\left[\frac{1}{r_{j}} \nabla_{\mathbf{r}_{i}} \cdot \nabla_{\mathbf{r}_{j}}+\frac{1}{r_{j}^{3}} \mathbf{r}_{j}^{\prime}\left(\mathbf{r}_{j} \cdot \nabla_{\mathbf{r}_{i}}\right) \nabla_{\mathbf{r}_{j}}\right] \\
& +\frac{1}{2} \sum_{i=1}^{n} \sum_{j>i}^{n} \frac{q_{i} q_{j}}{m_{i} m_{j}}\left[\frac{1}{r_{i j}} \nabla_{\mathbf{r}_{i}} \cdot \nabla_{\mathbf{r}_{j}}+\frac{1}{r_{i j}^{3}} \mathbf{r}_{i j}^{\prime}\left(\mathbf{r}_{i j} \cdot \nabla_{\mathbf{r}_{i}}\right) \nabla_{\mathbf{r}_{j}}\right],
\end{aligned}
$$

$$
\begin{gathered}
H_{\mathrm{ss}}=-\frac{8 \pi}{3} \sum_{i=1}^{n} \frac{q_{0} q_{i}}{m_{0} m_{i}} \mathbf{s}_{0} \cdot \mathbf{s}_{i} \delta\left(\mathbf{r}_{i}\right) \\
-\frac{8 \pi}{3} \sum_{i=1}^{n} \sum_{j>i}^{n} \frac{q_{i} q_{j}}{m_{i} m_{j}} \mathbf{s}_{i} \cdot \mathbf{s}_{j} \delta\left(\mathbf{r}_{i j}\right), \\
H_{\mathrm{a}}=-2 \pi \quad \sum_{i=1}^{n} \frac{q_{0} q_{i}}{m_{0} m_{i}}\left(\frac{3}{4}+\mathbf{s}_{0} \cdot \mathbf{s}_{i}\right) \delta\left(\mathbf{r}_{i}\right) \\
-2 \pi \sum_{\substack{i=1 \\
i, j}}^{n} \sum_{j>i}^{n} \frac{q_{i} q_{j}}{m_{i} m_{j}}\left(\frac{3}{4}+\mathbf{s}_{i} \cdot \mathbf{s}_{j}\right) \delta\left(\mathbf{r}_{i j}\right) .
\end{gathered}
$$

Here we have used the notation $\mathbf{s}_{i} \equiv \mathbf{S}_{i+1}$. Assuming our particular case of HPs and taking into account the indistinguishability of electrons the expectation values of the Darwin, spin-spin, and annihilation channel interactions can be written simply as

$$
\begin{gathered}
\left\langle H_{\mathrm{d}}\right\rangle=-\frac{\pi}{2}\left\langle\delta\left(\mathbf{r}_{1}\right)-2 \delta\left(\mathbf{r}_{2}\right)-4 \delta\left(\mathbf{r}_{12}\right)+2 \delta\left(\mathbf{r}_{23}\right)\right\rangle, \\
\left\langle H_{\mathrm{ss}}\right\rangle=2 \pi\left\langle\delta\left(\mathbf{r}_{23}\right)\right\rangle, \\
\left\langle H_{\mathrm{a}}\right\rangle=3 \pi\left\langle\delta\left(\mathbf{r}_{12}\right)\right\rangle .
\end{gathered}
$$

The details on evaluating matrix elements of various operators with basis functions (5) were presented elsewhere [40,42,53]. Here we will only mention a scheme that allows to significantly improve the convergence of expectation values that involve the Dirac $\delta$ functions. Such expectation values are needed not only in the calculations of the relativistic corrections to the energy, but also for the estimations of the decay rates, which will be considered later in this work. It is a known problem that expectation values of singular operators, such as the twoparticle $\delta$ function, usually exhibit rather poor convergence in variational calculations when compared to the expectation values of "well-behaved" operators. The main reason for this is the fact that the expectation values of singular operators are very sensitive to the local properties of the trial wave function. When a singular operator's expectation value is evaluated, the integration occurs only in a small region of space (essentially in some subspace). Hence, the wave function is sampled only locally in such an integration. At the same time, it is known that while the energy (as well as the expectation values of "wellbehaved," nonsingular operators) in the variational method is accurate to the second order, locally the wave function is accurate to the first order only. In other words, the local convergence of the wave function is significantly slower than the convergence of the energy (roughly speaking, one should expect twice fewer digits converged). This behavior is rather universal and independent of the basis set used. However, the 
properties of a particular basis may amplify this problem even further. In the case of Gaussians, the inability to satisfy the Kato cusp conditions usually makes the slower convergence of the two-particle $\delta$ functions even worse. In order to alleviate the problem Drachman [54] proposed to replace the local $\delta$ function operator with a global operator, which, for the exact wave function, would give the same expectation value. Since this operator is global the convergence of its expectation value is noticeably better as has been demonstrated in [55], where some generalizations of the above idea were considered. In this work we adopted the following identity from [54], which holds for the exact wave function:

$$
\begin{aligned}
\left\langle\psi\left|\tilde{\delta}\left(\mathbf{R}_{i j}\right)\right| \psi\right\rangle= & \frac{1}{2 \pi} \frac{M_{i} M_{j}}{M_{i}+M_{j}}\left[\left\langle\psi\left|\frac{2}{R_{i j}}(E-V)\right| \psi\right\rangle\right. \\
& \left.-\sum_{k=1}^{N} \frac{1}{M_{k}}\left\langle\nabla_{\mathbf{R}_{k}} \psi\left|\frac{1}{R_{i j}}\right| \nabla_{\mathbf{R}_{k}} \psi\right\rangle\right] .
\end{aligned}
$$

Here $E$ is the total nonrelativistic energy corresponding to state $\psi$, and $V$ is the potential energy operator. To distinguish the expectation values obtained using this identity from those computed directly, we use the tilde. In the internal coordinate frame, expression (25) acquires the following form:

$$
\begin{aligned}
\left\langle\psi\left|\tilde{\delta}\left(\mathbf{r}_{i j}\right)\right| \psi\right\rangle= & \frac{1}{2 \pi} \frac{1}{\operatorname{tr}\left[M J_{i j}\right]}\left[\left\langle\psi\left|\frac{2}{r_{i j}}(E-V)\right| \psi\right\rangle\right. \\
& \left.-\left\langle\nabla_{\mathbf{r}} \psi\left|\frac{1}{r_{i j}} \mathbf{M}\right| \nabla_{\mathbf{r}} \psi\right\rangle\right]
\end{aligned}
$$

where $\operatorname{tr}[\ldots]$ stands for the trace, $M$ is the mass matrix defined in (3), and $J_{i j}$ is a $n \times n$ matrix whose only nonzero elements are the following four ones: $\left(J_{i j}\right)_{i i}=\left(J_{i j}\right)_{j j}=1$, $\left(J_{i j}\right)_{i j}=\left(J_{i j}\right)_{j i}=-1$. For the case of the $\tilde{\delta}\left(\mathbf{r}_{i}\right)$ expectation value, one just needs to replace $J_{i j} \rightarrow J_{i i}\left(J_{i i}\right.$ matrix is defined such that the only nonzero element of it is $\left.\left(J_{i i}\right)_{i i}=1\right)$ and $r_{i j} \rightarrow r_{i}$ in the right-hand side of the above formula.

Despite being a stable bound system in nonrelativistic quantum mechanics, in reality HPs undergoes a decay due to the electron-positron annihilation, similar to the one in the positronium atom, Ps. It is possible to determine the rate of the HPs decay by computing the average square of the amplitude of the wave function at the electron-positron coalescence points and relating this value to the well studied decay rate of the positronium atom, i.e., using the relation

$$
\Gamma^{\mathrm{HPs}}=N_{e^{+}} N_{e^{-}} \frac{\left\langle\delta_{e^{+} e^{-}}\right\rangle^{\mathrm{HPs}}}{\left\langle\delta_{e^{+} e^{-}}\right\rangle^{\mathrm{Ps}}} \Gamma^{\mathrm{Ps}},
$$

where $N_{e^{+}}$and $N_{e^{-}}$are the numbers of positrons and electrons in the system. In general, the total decay rate of a system is the sum of $k$-photon annihilation rates,

$$
\Gamma=\sum_{k=0}^{\infty} w_{k} \Gamma_{k \gamma}
$$

where $w_{k}$ is the statistical weight of the spin state, which undergoes a particular $k$-photon annihilation. For the positronium atom both $\Gamma_{0 \gamma}$ and $\Gamma_{1 \gamma}$ are zeros, whereas for HPs they are extremely small in magnitude (the rate of $0 \gamma$ and $1 \gamma$ processes is proportional to $\alpha^{12} c / a_{0}$ and $\alpha^{8} c / a_{0}$, respectively; here $c$ is the speed of light and $a_{0}$ is the Bohr radius). The dominating components are $\Gamma_{2 \gamma} \propto \alpha^{4} c / a_{0}$ and $\Gamma_{3 \gamma} \propto \alpha^{5} c / a_{0}$. The two-photon decay takes place when an electron-positron pair is in the singlet state (the statistical weight of which is $w=1 / 4$ ), while the three-photon decay occurs in the triplet state $(w=3 / 4)$. The expressions for $\Gamma_{2 \gamma}$ and $\Gamma_{3 \gamma}$ in the positronium atom, including several leading radiative corrections, are known [56-58]. In this work, however, we must limit ourselves with the corrections proportional to $\alpha^{2} \ln \frac{1}{\alpha}$ in $\Gamma_{2 \gamma}^{\mathrm{Ps}}$ and $\alpha^{0}$ in $\Gamma_{3 \gamma}^{\mathrm{Ps}}$. This is because the expectation value of the electron-positron contact density in HPs available to us from calculations is purely nonrelativistic (i.e., does not include the leading correction of the order of $\alpha^{2}$ ) and, therefore, the right-hand side in expression (27) can only be accurate up to terms greater than $\alpha^{2}$. Using

$$
\begin{gathered}
\Gamma_{2 \gamma}^{\mathrm{Ps}}=4 \pi \frac{\alpha^{4} c}{a_{0}}\left\langle\delta_{e^{+} e^{-}}\right\rangle^{\mathrm{Ps}}\left[1-\left(5-\frac{\pi^{2}}{4}\right) \frac{\alpha}{\pi}+2 \alpha^{2} \ln \frac{1}{\alpha}+\cdots\right], \\
\Gamma_{3 \gamma}^{\mathrm{Ps}}=\frac{16}{9}\left(\pi^{2}-9\right) \frac{\alpha^{5} c}{a_{0}}\left\langle\delta_{\mathrm{e}^{+} \mathrm{e}^{-}}\right\rangle^{\mathrm{Ps}}[1+\cdots],
\end{gathered}
$$

and the known value $\left\langle\delta_{\mathrm{e}^{+} \mathrm{e}^{-}}\right\rangle^{\mathrm{Ps}}=1 /(8 \pi)$ the expression for the total electron-positron annihilation rate in HPs becomes

$$
\Gamma^{\mathrm{HPs}}=2 \pi \frac{\alpha^{4} c}{a_{0}}\left\langle\delta_{\mathrm{e}^{+} \mathrm{e}^{-}}\right\rangle^{\mathrm{HPs}}\left[1+\left(\frac{19 \pi}{12}-\frac{17}{\pi}\right) \alpha+2 \alpha^{2} \ln \frac{1}{\alpha}\right] .
$$

\section{COMPUTATIONAL DETAILS}

The choice of nonlinear variational parameters plays a crucial role in calculations that involve explicitly correlated basis functions. It is a particularly important subject when Gaussian functions are used. In fact, in calculations that require high accuracy it is the optimization of nonlinear parameters that consumes most of the computer time. Several approaches have been proposed to to deal with high computational demands [39,41,42,59-64]. In this work we have used an approach that combines a stochastic selection of the parameters $[41,42,59]$ with a direct optimization that uses the analytic energy gradient $[39,40,64]$. In the present calculations the basis set was grown from zero to 4000 functions. During this process, the basis was reoptimized a large number of times (essentially after adding each new subset of ten basis functions). In order to ensure high-numerical stability of the calculations we did not allow any severe linear dependencies among basis functions. This was done through monitoring overlaps of basis functions. Those changes of the nonlinear parameters that resulted in excessively high absolute value of an overlap (i.e., those that yielded $\mathrm{S}_{k l}>1-t$, where $t$ is some small threshold, which we set to be around 0.01) were automatically rejected. Such monitoring is a computationally inexpensive procedure and at the same time it is quite efficient. Our experience suggests that most of linear dependencies in the calculations of systems such as HPs appear as linear dependencies between two basis functions.

In the calculations we used standard double precision (64-bit) arithmetic until the basis size reached approximately 
TABLE I. Nonrelativistic energies and relativistic corrections for isotopologues of HPs. The tilde sign indicates that the expectation values of the corresponding operators were evaluated using relation (26). Values in parentheses represent estimates of the remaining uncertainty due to the finite size of the basis set used. At the bottom of the table we also show the nonrelativistic energies and relativistic corrections for isolated hydrogen-like atoms. All values are in atomic units.

\begin{tabular}{|c|c|c|c|c|c|c|c|c|}
\hline system & basis size & $\left\langle H_{\text {nonrel }}\right\rangle$ & $\left\langle H_{\mathrm{mv}}\right\rangle$ & $\left\langle\tilde{H}_{\mathrm{d}}\right\rangle$ & $\left\langle H_{\mathrm{oo}}\right\rangle$ & $\left\langle\tilde{H}_{\mathrm{ss}}\right\rangle$ & $\left\langle\tilde{H}_{\mathrm{a}}\right\rangle$ & $\left\langle H_{\text {nonrel }}+\alpha^{2} H_{\text {rel }}\right\rangle$ \\
\hline \multirow[t]{5}{*}{ HPs } & 500 & -0.788870347543 & -0.75613534 & 0.69384798 & -0.13496075 & 0.02739734 & 0.23085676 & -0.788867098892 \\
\hline & 1000 & -0.788870685002 & -0.75641409 & 0.69384828 & -0.13496084 & 0.02739837 & 0.23085702 & -0.788867451115 \\
\hline & 2000 & -0.788870709195 & -0.75659279 & 0.69384834 & -0.13496089 & 0.02739846 & 0.23085701 & -0.788867484819 \\
\hline & 3000 & -0.788870711910 & -0.75663266 & 0.69384834 & -0.13496088 & 0.02739847 & 0.23085702 & -0.788867489656 \\
\hline & 4000 & $-0.788870712244(200)$ & -0.75664884 & 0.69384834 & -0.13496088 & 0.02739847 & 0.23085702 & $-0.788867490851(1000)$ \\
\hline DPs & 4000 & $-0.789033601257(200)$ & -0.75739790 & 0.69431968 & -0.13468343 & 0.02742746 & 0.23087538 & $-0.789030377357(1000)$ \\
\hline TPs & 4000 & $-0.789087802858(200)$ & -0.75764728 & 0.69447657 & -0.13459104 & 0.02743712 & 0.23088149 & $-0.789084578123(1000)$ \\
\hline${ }^{\infty} \mathrm{HPs}$ & 4000 & $-0.789196766900(200)$ & -0.75814880 & 0.69479204 & -0.13440521 & 0.02745653 & 0.23089378 & $-0.789193540488(1000)$ \\
\hline $\operatorname{Ps}\left({ }^{1} S\right)$ & & -0.250000000000 & -0.07812500 & 0.12500000 & -0.12500000 & -0.25000000 & 0.00000000 & -0.250017473101 \\
\hline $\operatorname{Ps}\left({ }^{3} S\right)$ & & -0.250000000000 & -0.07812500 & 0.12500000 & -0.12500000 & 0.08333333 & 0.25000000 & -0.249986409811 \\
\hline $\mathrm{H}$ & & -0.499727839712 & -0.62364031 & 0.49918396 & -0.00054373 & & & -0.499734496135 \\
\hline $\mathrm{D}$ & & -0.499863815247 & -0.62431935 & 0.49959156 & -0.00027222 & & & -0.499870471667 \\
\hline $\mathrm{T}$ & & -0.499909056541 & -0.62454541 & 0.49972722 & -0.00018182 & & & -0.499915712961 \\
\hline${ }^{\infty} \mathrm{H}$ & & -0.500000000000 & -0.62500000 & 0.50000000 & 0.00000000 & & & -0.500006656419 \\
\hline
\end{tabular}

2500 functions. After that we switched to extended precision (80-bit). This was done to enable more efficient optimization of nonlinear parameters, which is very sensitive to the accuracy of the eigenvalues and eigenvectors obtained. Arithmetic operations with 80-bit precision, just like those performed with 64-bit precision, are hardware-accelerated in any x86compatible CPU found in most commodity computers today. While there is a certain performance penalty associated with the 80-bit precision, using it does not result in an enormous slow down of the calculations.

The optimization of nonlinear parameters in this work was performed only for the lightest isotopologue, HPs. Since the wave functions of DPs, TPs, and ${ }^{\infty}$ HPs are very close to that of HPs, the change can be effectively accounted for by readjusting the linear coefficients of the basis functions, i.e., by computing a new Hamiltonian matrix and solving an eigenvalue problem (which needs to be done only once) using the same basis set. Due to the smallness of the change of the wave function, such a time-saving simplification has essentially no effect on the accuracy of the calculations.

The vast majority of the computational time in the variational calculations of this study is spent on two tasks: the evaluation of the Hamiltonian and overlap matrix elements and the solution of the generalized eigenvalue problem (8). The first task can be easily and very effectively parallelized. The second one also allows a certain degree of parallelization (although the scalability is somewhat worse). Therefore, efficient algorithms that utilize parallelism in either shared memory or distributed memory environments can be developed. In our case all calculations have been carried out using 4-16 processes communicating via Message Passing Interface (MPI) protocol.

Due to the very extensive optimization of the nonlinear parameters, the generation of the basis set used in this work required several months of continuous computing. We stopped when the basis length reached 4000. However, if it becomes necessary in the future, the calculations can be easily resumed.

\section{RESULTS AND DISCUSSION}

In Table I we show the convergence of the nonrelativistic energy of HPs in terms of the number of basis functions. We also present the energies obtained with the final basis set for other isotopologues of the positronium hydride: DPs, TPs, and ${ }^{\infty}$ HPs. According to our estimates (which are based on studying the convergence patterns and extrapolating to the limit of an infinite basis set size) the accuracy of the nonrelativistic calculations in this work exceeds the previous best result [16] by nearly an order of magnitude in spite of a smaller number of basis functions used. In fact, the energy corresponding to the largest basis set of 5000 explicitly correlated Gaussians generated in work [16] was reached with only 2150 basis functions in this work. The better convergence is a result of a significantly more extensive optimization of the nonlinear parameters. It should be noted that the difference in the optimization quality becomes particularly pronounced when the basis size is large enough (several thousand functions), while for small basis sets the extra computational effort does not seem to be justified.

In addition to the nonrelativistic energies, in Table I we also show all components of the relativistic corrections and the total relativistic energies. When computing the latter quantity we used $\alpha=0.0072973525376$ for the value of the fine structure constant [37]. In general, the computed relativistic corrections agree with those obtained by Yan and Ho [13] using the Hylleraas-type basis. However, we have found that the value of the mass-velocity correction in work [13] is likely to contain an error. In the case of ${ }^{\infty}$ HPs (we use this isotopologue for comparison because not all of the recoil relativistic effects were calculated in [13]) we obtained $-4.03725 \times 10^{-5}$ a.u. for the expectation value of $H_{\mathrm{mv}}$ multiplied by $\alpha^{2}$, while Yan and Ho reported $-2.60410(30) \times 10^{-5}$ a.u. We believe our value is correct as with our computer code we have evaluated $\left\langle H_{\mathrm{mv}}\right\rangle$ in the case of several other atomic and molecular systems (including those containing positrons) for which independent results are available in the literature, and found no discrepancies in any of those cases. 
TABLE II. Binding energies (in eV) for isotopologues of HPs obtained both in nonrelativistic calculations and after the inclusion of the relativistic corrections with the largest basis of 4000 functions. Values in parentheses represent estimates of the remaining uncertainty due to the finite size of the basis set used.

\begin{tabular}{lcc}
\hline \hline system & nonrel. & rel. \\
\hline HPs & $1.065131730(4)$ & $1.064387474(50)$ \\
DPs & $1.065864083(4)$ & $1.065119758(50)$ \\
TPs & $1.066107905(4)$ & $1.065363558(50)$ \\
${ }^{\infty} \mathrm{HPs}$ & $1.066598270(4)$ & $1.065853877(50)$ \\
\hline \hline
\end{tabular}

At the bottom of Table I we provide the values of the total energies and relativistic corrections for isolated hydrogen-like atoms. Combined in appropriate pairs these can be used to compare them with the corresponding values for the HPs isotopologues.

The knowledge of the total binding energies of the HPs isotopologues as well as its subsystems ( $\mathrm{H}$ and Ps atoms) allows the determination of the binding energies. The cor- responding data is given in Table II. The inclusion of the relativistic corrections has a very small effect on the binding energies (less than $0.1 \%$ change). This effect is almost $10 \mathrm{x}$ larger in magnitude than the shift of the total energy due to the inclusion of the relativistic corrections. Therefore, most of the change in the binding energy is due to the relativistic effects in separated $\mathrm{H}$ and Ps atoms. The relativistic effects in HPs and in the isolated $\mathrm{H}$ and Ps atoms do not cancel out significantly as takes place in some weakly bound molecules. Indeed, while the positronium hydride structure is consistent with that of a molecule (rather than an atom), the binding and the interaction of the electrons cannot be considered weak.

In Table III we present the expectation values of various quantities. These include the powers of the interparticle distances, two-particle Dirac $\delta$ functions $\delta\left(\mathbf{r}_{i j}\right) \equiv \delta\left(x_{i}-\right.$ $\left.x_{j}\right) \delta\left(y_{i}-y_{j}\right) \delta\left(z_{i}-z_{j}\right)$, products of two Dirac $\delta$ functions $\delta\left(\mathbf{r}_{i j}\right) \delta\left(\mathbf{r}_{l m}\right)$ and $\delta\left(\mathbf{r}_{i j}\right) \delta\left(\mathbf{r}_{j k}\right)$ (the latter determine the threeparticle coalescence probabilities), and the product of three Dirac $\delta$ functions, $\delta\left(\mathbf{r}_{i j}\right) \delta\left(\mathbf{r}_{j k}\right) \delta\left(\mathbf{r}_{k l}\right)$, which in the case of a four-particle system, such as HPs, is equal to the value of the wave function square at the origin. For convenience of the

TABLE III. Expectation values of various powers of interparticle distances and the Dirac $\delta$ functions. For the expectation values of a single $\delta$ function we show the results obtained in both the direct calculations and using relation (26). The latter ones are marked with a tilde. Indices $a$ and $b$ in some double $\delta$ functions emphasize that the two electrons are different. All values are in atomic units.

\begin{tabular}{|c|c|c|c|c|c|c|c|c|c|}
\hline systen & basis size & $\left\langle 1 / r_{\mathrm{H}^{+} e^{+}}^{2}\right\rangle$ & $\left\langle 1 / r_{e^{+} e^{-}}^{2}\right\rangle$ & $\left\langle 1 / r_{\mathrm{H}^{+} e^{-}}^{2}\right\rangle$ & $\left\langle 1 / r_{e^{-} e^{-}}^{2}\right\rangle$ & $\left\langle 1 / r_{\mathrm{H}^{+} e^{+}}\right\rangle$ & $\left\langle 1 / r_{e^{+}} e^{-}\right\rangle$ & $\left\langle 1 / r_{\mathrm{H}^{+} e^{-}}\right\rangle$ & $\left\langle 1 / r_{e^{-} e^{-}}\right\rangle$ \\
\hline \multirow[t]{5}{*}{ HPs } & 500 & 0.172014772 & 0.349071114 & 1.205649513 & 0.213648294 & 0.347301925 & 0.418428418 & 0.729258284 & 0.370330922 \\
\hline & 1000 & 0.172013641 & 0.349072614 & 1.205651819 & 0.213646523 & 0.347301530 & 0.418428480 & 0.729258149 & 0.370 \\
\hline & 2000 & 0.172013540 & 0.349072759 & 1.205652123 & 0.213646371 & 0.347301497 & 0.418428492 & 0.729258147 & 0.370330354 \\
\hline & 3000 & 172013541 & 0.349072777 & 1.205652143 & 0.213646366 & 0.347301507 & 0.418428498 & 0.729258147 & 0.370 \\
\hline & 4000 & 172013540 & 0.349072780 & 1.205652147 & 0.213646365 & 0.347301507 & 0.418428498 & 258148 & 0.370 \\
\hline DPs & 4000 & 088169 & 0.349108392 & 1.206359185 & 0.213777865 & 0.347381564 & 0.4184 & 0.72 & 0.37 \\
\hline TPs & 000 & 113007 & 0.349120245 & 1.206594493 & 0.213821637 & 0.347408203 & 0.4184 & 0.729558301 & 0.370 \\
\hline${ }^{\infty}$ HPs & 4000 & 0.172162946 & 0.349144077 & 1.207067607 & 0.213909656 & 0.347461760 & 0.418496096 & 0.729708964 & 0.370554828 \\
\hline \multirow{6}{*}{$\begin{array}{l}\text { system } \\
\text { HPs }\end{array}$} & basis size & & & $\left\langle r_{\mathrm{H}^{+} e^{-}}\right\rangle$ & $\left\langle r_{e^{-}} e^{-}\right\rangle$ & & & & \\
\hline & 500 & 3.663490430 & 3.481169368 & 2.313152927 & 3.577005779 & 16.271935302 & 15.593396345 & 7.824650807 & 15.895655820 \\
\hline & 1000 & 3.663501879 & 3.481175784 & 2.313161069 & 3.577021997 & 16.272155569 & 15.593537619 & 7.824794250 & 938518 \\
\hline & 2000 & 3.663503158 & 3.481176236 & 2.313161605 & 3.577023204 & 16.272180014 & 15.5935 & 7.824804450 & 0551 \\
\hline & 3000 & 3.663502763 & 3.481176137 & 2.313161605 & 3.5770 & 16.272175247 & 15.593 & 7.824805150 & 9702 \\
\hline & 4000 & 3.6635 & & 2.313161609 & 3.5770 & 16.272175401 & 15.593 & 7.824805250 & 9906 \\
\hline DPs & 4000 & & 3.480724774 & 2.312344283 & 3.5759 & $16.26 ?$ & 15.588 & 7.818 & 6099 \\
\hline TPs & 4000 & 3.6622 & 3.4805 & 2.3120724 & 3.5755 & 16.260 & 15.587 & 7.816 & 2508 \\
\hline${ }^{\infty} \mathrm{HPs}$ & 4000 & 3.661625055 & 3.480272821 & 2.311525968 & 3.574788230 & 16.254539620 & 15.584279135 & 7.813052338 & 15.875482712 \\
\hline & basis size & & $\left\langle\delta_{e^{+} e^{-}}\right\rangle$ & $\left\langle\delta_{\mathrm{H}^{+} e^{-}}\right\rangle$ & & & & & \\
\hline \multirow[t]{5}{*}{ HPs } & 500 & 0.001638389 & 0.024458439 & 0.176894912 & 0.004382960 & 0.001622696 & 0.024494663 & 0.177041115 & 0.004360422 \\
\hline & 1000 & 0.001 & & & 761 & & 690 & 0.17 & 586 \\
\hline & 2000 & 0.00 & 0.0244 & 0 . & 0.004 & 0.00 & 690 & 0.177 & 0.00 \\
\hline & 3000 & 0.001 & 0.0244 & 0.177033 & 0.004 & 0.00 & 690 & 0.177 & 0.00 \\
\hline & 4000 & 0.00 & 0.02 & $0.177 \mathrm{C}$ & 0.004 & 903 & 690 & 0.177 & 0.00 \\
\hline DPs & 4000 & 0.00 & 0.024 & 0.1771 & 0.004 & 745 & 639 & 0.177 & 5216 \\
\hline TPs & 4000 & 0.00 & 0.024 & 0.1772 & 0.004 & 0.00 & 287 & 0.177 & 753 \\
\hline${ }^{\infty} \mathrm{HPs}$ & 4000 & 0.001624841 & 0.024497366 & 0.177340118 & 0.004370403 & 0.001624589 & 0.024498591 & 0.177344131 & 0.004369843 \\
\hline \multicolumn{10}{|c|}{ system basis size } \\
\hline \multirow[t]{5}{*}{ HPs } & 500 & $8.7403 \times 10^{-4}$ & $3.1225 \times 10^{-5}$ & $6.2365 \times 10^{-3}$ & $7.5321 \times 10^{-3}$ & $3.7460 \times 10^{-4}$ & $1.9180 \times 10^{-4^{b}}$ & & \\
\hline & 1000 & $8.5986 \times 10^{-4}$ & $3.1582 \times 10^{-5}$ & $6.3212 \times 10^{-3}$ & $7.5334 \times 10^{-3}$ & $3.7147 \times 10^{-4}$ & $1.9038 \times 10^{-4}$ & & \\
\hline & 2000 & $8.5038 \times 10^{-4}$ & $3.1749 \times 10^{-5}$ & $6.3509 \times 10^{-3}$ & $7.5404 \times 10^{-3}$ & $3.6961 \times 10^{-4}$ & $1.8898 \times 10^{-4}$ & & \\
\hline & 3000 & $8.4933 \times 10^{-4}$ & $3.1856 \times 10^{-5}$ & $6.3597 \times 10^{-3}$ & $7.5406 \times 10^{-3}$ & $3.6907 \times 10^{-4}$ & $1.8782 \times 10^{-4}$ & & \\
\hline & 4000 & $8.4725 \times 10^{-4}$ & $3.1909 \times 10^{-5}$ & $6.3646 \times 10^{-3}$ & $7.5432 \times 10^{-3}$ & $3.6887 \times 10^{-4}$ & $1.8738 \times 10^{-4}$ & & \\
\hline DPs & 4000 & $8.4819 \times 10^{-4}$ & $3.1947 \times 10^{-5}$ & $6.3701 \times 10^{-3}$ & $7.5589 \times 10^{-3}$ & $3.6918 \times 10^{-4}$ & $1.8772 \times 10^{-4}$ & & \\
\hline TPs & 4000 & $8.4850 \times 10^{-4}$ & $3.1960 \times 10^{-5}$ & $6.3719 \times 10^{-3}$ & $7.5641 \times 10^{-3}$ & $3.6928 \times 10^{-4}$ & $1.8783 \times 10^{-4}$ & & \\
\hline${ }^{\infty}$ HPs & 4000 & $8.4913 \times 10^{-4}$ & $3.1985 \times 10^{-5}$ & $6.3756 \times 10^{-3}$ & $7.5746 \times 10^{-3}$ & $3.6948 \times 10^{-4}$ & $1.8806 \times 10^{-4}$ & & \\
\hline
\end{tabular}


reader, in Table III instead of pseudoparticle indices $(1,2$, or 3$)$ we use subscripts indicating the actual particles, for example $\mathbf{r}_{\mathrm{H}^{+} e^{+}} \equiv \mathbf{r}_{1}$ or $\mathbf{r}_{e^{+} e^{-}} \equiv \mathbf{r}_{23}$. As one can see from the data in Table III, the convergence of the nonsingular operators such as powers of the interparticle distances is similar (just slightly worse) to that of the nonrelativistic energy. The situation is different for the singular operators. The expectation values of the Dirac $\delta$ functions computed directly, as expected, show a substantially lower level of convergence. The convergence gets worse for the products of two Dirac $\delta$ functions; and for the product of three $\delta$ functions the accuracy probably does not exceed three decimal figures. As was explained in Sec. II, in addition to the fact that, unlike the energy, the wave function in the variational method is accurate only to the first order, we face the problem of a somewhat poor description of the cusp region by the Gaussian-type basis. In contrast to the direct evaluation of the expectation values of the pair $\delta$ functions, the use of relation (26) noticeably improves the convergence. Unfortunately, such a transformation is likely to be possible only for the expectation values of $\delta\left(\mathbf{r}_{i j}\right)$. More singular operators, such as the products of two and more $\delta$ functions will probably require some matrix elements that are too difficult to evaluate.

Next, we use the expectation values of the pair Dirac $\delta$ functions with formula (31) to determine the rates of the electron-positron annihilation in HPs isotopologues. The computed values are shown in Table IV. The numeric uncertainties (due to the finiteness of the basis set used) of these values are very small and do not appear in the significant figures shown in Table IV. Much larger is the uncertainty due to the missing $\alpha^{2}$ and higher-order terms
TABLE IV. Electron-positron annihilation rates (in $\mathrm{sec}^{-1}$ ) for HPs isotopologues.

\begin{tabular}{lc}
\hline \hline system & $\Gamma$ \\
\hline HPs & $2.465156 \times 10^{9}$ \\
DPs & $2.465352 \times 10^{9}$ \\
TPs & $2.465418 \times 10^{9}$ \\
${ }^{\infty} \mathrm{HPs}$ & $2.465549 \times 10^{9}$ \\
\hline \hline
\end{tabular}

in formula (31), which we roughly estimate to be of the order of $0.0003 \times 10^{9} \mathrm{~s}^{-1}$.

In summary, high-accuracy variational calculations of the ground state of positronium hydride and its isotopologues have been performed using a variational expansion in terms of explicitly correlated Gaussian basis functions. A new upper bound to the nonrelativistic energy has been obtained and leading relativistic corrections have been computed. In this work we have improved the accuracy of binding energies, various expectation values, and electron-positron annihilation rates. We also corrected and expanded the results of the only previous study that considered relativistic effects in HPs.

\section{ACKNOWLEDGMENTS}

The authors thank University of Arizona Research Computing Services for an opportunity to use an SGI Altix ICE 8200 computer cluster, where a part of the calculations was performed.
[1] E. A. Hylleraas and A. Ore, Phys. Rev. 71, 493 (1947).

[2] A. Ore, Phys. Rev. 83, 665 (1951).

[3] C. F. Lebeda and D. M. Schrader, Phys. Rev. 178, 24 (1969).

[4] Y. K. Ho, Phys. Rev. A 34, 609 (1986).

[5] T. Yoshida and G. Miyako, Phys. Rev. A 54, 4571 (1996).

[6] A. M. Frolov and V. H. Smith, Phys. Rev. A 55, 2662 (1997).

[7] A. M. Frolov and V. H. Smith, Phys. Rev. A 56, 2417 (1997).

[8] D. Bressanini, M. Mella, and G. Morosi, Phys. Rev. A 57, 1678 (1998).

[9] N. Jiang and D. M. Schrader, J. Chem. Phys. 109, 9430 (1998).

[10] J. Usukura, K. Varga, and Y. Suzuki, Phys. Rev. A 58, 1918 (1998).

[11] K. Strasburger and H. Chojnacki, J. Chem. Phys. 108, 3218 (1998).

[12] K. Varga, Phys. Rev. Lett. 83, 5471 (1999).

[13] Z.-C. Yan and Y. K. Ho, Phys. Rev. A 60, 5098 (1999).

[14] S. L. Saito, J. Chem. Phys. 118, 1714 (2003).

[15] J. Mitroy, Phys. Rev. A 73, 054502 (2006).

[16] S. Bubin and L. Adamowicz, Phys. Rev. A 74, 052502 (2006).

[17] M. W. J. Bromley, J. Mitroy, and K. Varga, Phys. Rev. A 75, 062505 (2007)

[18] J. Mitroy and M. W. J. Bromley, Phys. Rev. Lett. 98, 063401 (2007).
[19] Z.-C. Yan and Y. K. Ho, Phys. Rev. A 78, 012711 (2008).

[20] D. M. Schrader, F. M. Jacobsen, N.-P. Frandsen, and U. Mikkelsen, Phys. Rev. Lett. 69, 57 (1992).

[21] D. B. Cassidy and A. P. Mills, Nature (London) 449, 195 (2007).

[22] D. B. Cassidy, S. H. M. Deng, and A. P. Mills, Phys. Rev. A 76, 062511 (2007)

[23] C. Surko, Nature (London) 449, 153 (2007).

[24] C. Gahn, G. D. Tsakiris, G. Pretzler, K. J. Witte, C. Delfin, C.-G. Wahlström, and D. Habs, Appl. Phys. Lett. 77, 2662 (2000).

[25] H. Chen, S. C. Wilks, J. D. Bonlie, E. P. Liang, J. Myatt, D. F. Price, D. D. Meyerhofer, and P. Beiersdorfer, Phys. Rev. Lett. 102, 105001 (2009).

[26] K. Varga, J. Usukura, and Y. Suzuki, Phys. Rev. Lett. 80, 1876 (1998).

[27] D. Bressanini, M. Mella, and G. Morosi, Phys. Rev. A 55, 200 (1997).

[28] E. Armour, J.-M. Richard, and K. Varga, Phys. Rep. 413, 1 (2005).

[29] T. Rebane, Phys. At. Nucl. 72, 55 (2009).

[30] J.-M. Richard, Phys. Rev. A 49, 3573 (1994).

[31] J. Mitroy and S. A. Novikov, Phys. Rev. A 70, 032511 (2004).

[32] K. Varga, S. Fleck, and J. M. Richard, Europhys. Lett. 37, 183 (1997) 
[33] K. Strasburger, J. Phys. B 37, 4483 (2004).

[34] M. Amoretti et al., Nature (London) 419, 456 (2002).

[35] G. Gabrielse et al., (ATRAP Collaboration), Phys. Rev. Lett. 89, 213401 (2002).

[36] D. K. Gridnev and C. Greiner, Phys. Rev. Lett. 94, 223402 (2005).

[37] CODATA 2006 Recommended values of the fundamental physical constants, available at [http://physics.nist.gov/cuu].

[38] M. Cafiero, S. Bubin, and L. Adamowicz, Phys. Chem. Chem. Phys. 5, 1491 (2003).

[39] S. Bubin, M. Cafiero, and L. Adamowicz, Adv. Chem. Phys. 131, 377 (2005).

[40] S. Bubin and L. Adamowicz, J. Chem. Phys. 124, 224317 (2006).

[41] K. Varga and Y. Suzuki, Phys. Rev. C 52, 2885 (1995).

[42] Y. Suzuki and K. Varga, Stochastic Variational Approach to Quantum-Mechanical Few-Body Problems, Lecture Notes in Physics (Springer-Verlag, Berlin, 1998).

[43] J. Komasa, W. Cencek, and J. Rychlewski, Phys. Rev. A 52, 4500 (1995).

[44] J. Mitroy, Phys. Rev. A 70, 024502 (2004).

[45] S. Bubin and L. Adamowicz, J. Chem. Phys. 121, 6249 (2004).

[46] S. Bubin, M. Stanke, D. Kędziera, and L. Adamowicz, Phys. Rev. A 75, 062504 (2007).

[47] M. Stanke, D. Kędziera, S. Bubin, and L. Adamowicz, Phys. Rev. Lett. 99, 043001 (2007).

[48] W. Cencek and K. Szalewicz, Int. J. Quantum Chem. 108, 2191 (2008).
[49] M. Puchalski and A. Czarnecki, Phys. Rev. Lett. 101, 183001 (2008).

[50] S. Bubin and L. Adamowicz, Phys. Rev. A 83, 022505 (2011).

[51] H. A. Bethe and E. E. Salpeter, Quantum Mechanics of Oneand Two-Electron Atoms (Plenum, New York, 1977).

[52] V. B. Berestetskii, E. M. Lifshitz, and L. P. Pitaevskii, Kvantovaya Elektrodinamika (Quantum Electrodynamics) (Nauka, Moscow, 1989), in Russian.

[53] S. Bubin, M. Stanke, D. Kędziera, and L. Adamowicz, Phys. Rev. A 76, 022512 (2007).

[54] R. J. Drachman, J. Phys. B 14, 2733 (1981).

[55] K. Pachucki, W. Cencek, and J. Komasa, J. Chem. Phys. 122, 184101 (2005).

[56] G. S. Adkins, R. N. Fell, and J. Sapirstein, Phys. Rev. Lett. 84, 5086 (2000).

[57] A. Czarnecki, K. Melnikov, and A. Yelkhovsky, Phys. Rev. A 61, 052502 (2000).

[58] B. A. Kniehl, A. V. Kotikov, and O. L. Veretin, Phys. Rev. A 80, 052501 (2009).

[59] V. I. Kukulin and V. M. Krasnopol'sky, J. Phys. G 3, 795 (1977).

[60] S. A. Alexander, H. J. Monkhorst, and K. Szalewicz, J. Chem. Phys. 85, 5821 (1986).

[61] S. A. Alexander, H. J. Monkhorst, and K. Szalewicz, J. Chem. Phys. 87, 3976 (1987).

[62] P. M. Kozlowski and L. Adamowicz, J. Chem. Phys. 95, 6681 (1991).

[63] P. M. Kozlowski and L. Adamowicz, J. Chem. Phys. 96, 9013 (1992).

[64] D. B. Kinghorn, Int. J. Quantum Chem. 57, 141 (1996). 